\title{
Towards a narrative theory of Virtual Reality
}

\author{
Sandy Louchart, Ruth Aylett \\ The Centre for Virtual Environments, \\ University of Salford, Salford, \\ Manchester M5 4WT \\ \{S.Louchart, R.S.Aylett\}@Salford.ac.uk
}

\begin{abstract}
Virtual Reality (VR), by its nature and characteristics, is of specific interest to the AI community, particularly in the domains of Storytelling and Intelligent Characters. We argue that VR must be considered a particular narrative medium alongside Theatre, Literature or Cinema. This paper reviews relevant work in narrative theory from Plato onwards, including the work and theories of literary critics [1], cinema critics $[2,3,4]$ and theatrical dramaturges [5], and analyses the specific characteristics of VR relevant to this theory. Less studied media such as Live Role Playing Games, improvisational drama and participatory drama are also considered. Finally, this document argues for a participatory process-oriented narrative, with particular attention to the specificities and particularities of stories and their possible representation, adapted to the narrative medium Virtual Reality.
\end{abstract}

Keywords \{Virtual Reality, Narrative theory, Storytelling, Interactivity, User experience, Emergent narrative\} 


\section{INTRODUCTION}

\subsection{A need for investigation}

Virtual Reality (VR) has now progressed beyond the simple act of technical discovery towards a valid entertainment medium in its own right. A systematic exploration of the potentials, possibilities, advantages and constraints of this technology now needs to be carried out in relation to different types of functionality and application. Given that VR is of specific interest to the AI community in the domains of Storytelling and Intelligent Characters, these are particularly relevant areas for research.

Just as narrative in film was originally seen through the lens of narrative in the novel, so there is a tendency to consider narrative in VR in relation to film or television, or to even earlier narrative theories. Despite some very influential work based on this approach $[6,7,8,9,10,11,12,13]$, a thorough investigation of the nature of VR itself should be conducted in order to identify narrative forms and means of communication specific to this medium.

\subsection{VR as a narrative medium}

We argue that VR should be considered as a specific narrative medium alongside other narrative forms such as Theatre, Literature or Cinema. Each of these presents particularities that differentiate them from each other and determines their relative narrative forms, means of communication and displays of content in relation to story. A story is not told or shown in the same way according to the medium in which it is displayed, nor is its content or its intensity the same. The very different nature of media means that a narrative has either to be told or shown in different ways, varying 
the intensity of different aspects or parts of the content in order to achieve a satisfying effect on the person(s) to whom the narrative is communicated or displayed. The recent cinematic adaptation of "The Lord of the Rings" [14] illustrates this point, differing as it did in a number of respects from the original text, reflecting for example the more external visual perspective of film as against the internal charactercentred commentary of a novel. What is possible in a novel is not obviously realisable in a movie picture and vice versa. By their characteristics, narrative media generate different narrative forms that allow them to transmit the narrative in the most efficient way.

Virtual Reality, as a narrative medium, through its interactivity and other particularities, presents characteristics that none of the previously mentioned narrative forms usually possess, and should be recognised as such.

\subsection{Narrative as a dynamic process}

It is apparent that narrative theories have been heavily influenced by the idea that narrative must be authored. The relevant works and theories of Greek philosophy [15, $16]$, literary critics $[1,17]$, cinema critics $[2,3,4]$ and classic theatrical dramaturges, all converge towards an authorial view on narrative. However, characteristic of VR is that the role of the subject to whom the narrative is communicated is, in terms of interaction, "active" in the unfolding of the narrative as opposed to its "passive" role in most of other classical narrative media. Such distinction between spectator and user implicates that a differentiation must also be made between authorial and interactive approaches to narrative. On one hand, narrative is seen as an artefact that can be studied, involving non-interactive spectators, whereas, on the other hand, it could be 
perceived as the dynamic process resulting from the interaction between characters and its impact on the user (the 'Storification' process).

Those two distinctive approaches should be thoroughly considered in the elaboration of specific narrative forms and theories proper to VR.

\section{VR AS A VALID NARRATIVE FORM}

Narrative media such as literature, theatre, cinema or oral storytelling have attracted the attention and effort of an important number of authors. VR researchers have primarily focused on its technological capabilities, while in comparison to the previously mentioned narrative media, little account has been given of theoretical concerns. If evidence of differences between VR and other media justifies differentiation in narrative theory, an obvious approach is a comparative analysis. Analysing these differences should then provide us with valid arguments in favour of the recognition of VR as a narrative medium. Such a comparative approach requires careful attention to relevant theories, authors and discussions.

\subsection{Comparative considerations}

To make a comparison between Cinema, Theatre, Literature and VR supposes a set of comparative dimensions. We propose those of Contingency, Presence, Interactivity and Narrative Representation. By contingency, we mean how far the time and space of the narrative is contingent on real time and space; by presence how far the spectator/user physically shares the time and space of the narrative; by interactivity how far they interact with the story process and by narrative representation the characteristic form of narrative in the medium. This analysis will firstly demonstrate 
why VR should be recognised as a narrative form, but will also provide us with a set of factors and parameters specific to the application of VR. A particular attention to these factors should then contribute to the elaboration of a narrative theory specific and proper to VR.

Considering narrative representation first, it is clear that the format of the book is very different from a computer application, a cinema screen or a theatre stage. Novels largely deliver the story in such a way that the audience has to proceed to a mental representation of the narrative in order to image and imagine it, whereas, VR, Cinema and Theatre directly provide a visual form for the narrative.

Time and space considerations also vary with the media. Here one can distinguish between the time and space of narrative construction, of the narrative itself, and of the presentation of the narrative. Literature and Cinema are able to manipulate the time and space of the narrative very flexibly, while VR displays in real time, tying it very strongly to a specific space and time. These constraints are linked to the very nature of the medium, which lies in immersion and believability. A VR user would experience rapid and repeated travel from location to location and playing with time constraints as loss of control. A novel or film does not offer this sort of control in the first place. The narrative of theatre also takes place substantially in real time and a defined location, and here an episodic structure and the concept of 'off-stage' activity are used to produce some sense of temporal and spatial richness. 
Real time also brings certain constraints on the dramatic intensity of any narrative. To be of interest to an audience, a narrative displayed in real time must be either multiple, interactive or exceptionally rich in dramatic features. Real time is in fact incompatible with certain narrative forms such as Literature or Cinema. From an authorial point of view, it would imply the author writing, telling and displaying the story at the same time as the reader is reading or viewing it. From a reader or spectator's perspective, it would mean that narrative time was exactly that needed to read the book or the feature length of the movie. While real time in literature is actually impossible (the author would have to know how long it would take every reader to finish the book for instance), it is theoretically possible to direct a movie that could achieve, at screening, a simulation of real time for the spectator. However, this would only be valid from the spectator's perspective given that the action certainly does not actually happen at the moment it is displayed.

Theatre in its different forms, classic and modern, can display a narrative in real time and keep the audience interested, through the use of dramatically very rich narratives (classic) or by allowing narrative space for interaction between actors and what can be considered as "spectators/actors" [5]. However, it is important to note that, from an authorial point of view, classic theatre still does not present an exact representation of real time since the lines pronounced by the actors have been written beforehand. If the narrative time of a play obeys real-time constraints, this is not true of the narrative communicated, unless it is improvised. 
Theatre seems to be the only narrative medium that actually allows the spectator to be physically present at either, the elaboration, in the particular case of improvisational theatre, or the representation or display of a narrative. Literature; where the narrative representation is mental, and cinema; where the narrative representation doesn't physically happens in front of the spectators but months before the screening and on different locations, can be regarded by the spectators, from the perspective of narrative representation, as not being physically present in the same sense as theatre is. VR presents a certain challenge in assessing the presence of the user with respect to narrative representations in the sense that, as in Cinema, the users are not physically in the presence of the actors, but on the other hand, have more possibilities of interaction with the actors than in any other medium. The virtual presence of the actors, through the immersion VR generates, is actually of greater value from the perspective of interaction than their physical presence in classic theatre.

\subsection{Conclusion}

In Figure 1 we summarise the differences between VR and Theatre, Cinema or Literature VR, with its real time interaction, potentially offers high entertainment values. However, time and space constraints appear to be much more restrictive within VR than with the other narrative media considered. These arguments corroborate our earlier expressed view of a need for distinction and differentiation of the medium of VR as a narrative form in its own right. They also entitle us to submit VR to a thorough appraisal of narrative characteristics and compliance with existing narrative theories. 


\begin{tabular}{|c|c|c|c|c|}
\hline & Cinema & Theatre & Literature & VR \\
\hline $\begin{array}{l}\text { Contingency } \\
\text { on time and } \\
\text { space }\end{array}$ & Low & Medium & Low & Strong \\
\hline $\begin{array}{l}\text { Narrative } \\
\text { Representation }\end{array}$ & Visual & Visual & Mental & Visual \\
\hline Presence & Not physical & Physical & Not physical & $\begin{array}{l}\text { Not physical } \\
\text { but immersive }\end{array}$ \\
\hline Interactivity & No & $\begin{array}{l}\text { No / Yes in the } \\
\text { case of } \\
\text { interactive } \\
\text { theatre }\end{array}$ & No & Yes \\
\hline
\end{tabular}

Fig1. Comparative table of different narrative forms

\section{AN APPROACH TOWARDS A NARRATIVE THEORY OF VR}

\subsection{Introduction}

In studying narrative theories that have been developed over the years in cinematic, theatrical or literature research, one predictably finds that narrative theories have drawn upon each other to reach specific goals. Our starting point was to consider every narrative theory that presents any relevant aspect for a narrative definition of VR. However, assessing narrative theories according to their relevance to a narrative approach to VR proves to be fairly challenging. One big issue lies in finding a 
common ground between theories so that they can be considered, analysed and compared. If a comparative approach to the characteristics of different media appeared reasonable, a similar approach to narrative theories seems much more questionable. The spectrum of abstraction on which they rely is such that, for instance, a direct comparison between Aristotelian and Structuralist narrative considerations would find little of use for constructing a narrative theory particular to VR.

Thus we confine ourselves to considering the relevance of each separately to a theory for VR. We start with concepts from Plato's [15] high-level approach to narrative, also considered by Bordwell [2]. We apply the Platonic categories of "Diegesis" (the poet directly addresses the audience), and "Mimesis", (the poet addresses the audience through the use of characters) both to the narrative theories under consideration and to the various media, including VR. We locate Diegetic theories and narrative forms as "telling", in the tradition of oral storytelling, original Greek drama (at least the chorus) and substantially in the novel, and Mimetic forms and theories as "showing", as present in the forms of theatre or cinema. Such a categorisation allows us to consider narrative as a representation, a structure or a process [2]. The visual aspects of VR may suggest that we should give more priority to mimetic considerations as against diegetic ones. However potentially both can make a positive contribution, so we consider them equally. 


\subsection{Mimetic and Diegetic considerations and VR}

The AI community in the last few years has been strongly influenced by the Aristotelian approach to narrative and has recently produced significant work based on those conceptions $[6,7,8,9,10,11,12,13]$. However, Aristotle's plot centred approach [16] does not include interactivity between the author and the user as a possible factor or component of the narrative and this makes it hard to apply to VR without serious modification (hence Mateas describes his approach as 'neoAristotlean'). A plot centred approach conflicts with the freedom VR potentially offers to the user and can therefore be very restrictive. In order to reconcile interactivity and narrative while still providing the user with a satisfactory level of freedom within a 3D environment, Aylett as well as Nath [18, 19], argue for the consideration of a character based narrative form. This presents the double advantage for the user of, on the one hand, taking part in a unique experience, and on the other, acting freely without the constraints imposed by a plot centred approach.

Theatre and Cinema clearly work largely from a mimetic perspective, sharing a particular awareness of the spectator's visual engagement. Cinema and film theorists have added to the general Aristotelian conception of mimesis, for example by including the conception of narration from different perspectives in order to emphasise dramatic structure. The camera can then be thought of as "an observer ideally mobile in space and time" [4] or an invisible observer. However Bordwell [2] argues that these approaches only partially cover the narrational roles of other film techniques. 
These theories have had a substantial impact on technical aspects of cinematography through the use of particular camera angles and positions, but their contribution to the theory sought for VR is more problematic. In Cinema, the camera is under authorial control, so that the ideal observer it represents is in some sense the narrator. In VR, the camera is identified with the user, and removing their control over it directly contradicts the freedom to move and look that is one of the major defining characteristics of the medium. In this sense, VR moves beyond mimesis - 'showing' with its implication of direction, to 'experiencing'. Thus though both Cinema and VR share a synthetic visual aspect there are fundamental differences between them which make the narrative theory of film much less useful than one might have assumed.

Given that Aristotle gives little theoretical weight to the role of emotion in narrative (as distinct from its emotional impact upon the spectator), it is not surprising that the subsequent theories already mentioned do not pay any particular attention to emotion and its values. It is now believed that emotions play an important role in human cognition and are a major factor in the establishment of believability [20]. A narrative theory for VR must encompass the emotional contribution to believability, which contributes towards providing the user with a unique immersive experience. Eisenstein's expressionist approach [4] was to regard narration as making manifest some essential emotional quality of the story [2]. With the aim of a satisfactory user experience in mind, this expressionist narrative conception might be included in the consideration of a narrative model proper to VR. 
The narrative theories advanced by the Russian Formalists, the French structuralists and Heath's philosophical approach all have their roots in linguistics and the use of language, and so it seems reasonable to think of them as inclined towards a diegetic approach. Eichenbaum [21] viewed film shots as linked into phrases and sentences; Tynianov [21] sought for language structure in cinematic equivalents. For both - and for the Russian formalist movement - language and the linguistic represented the basis of cinema and of narrative in general. The Russian formalists however, in their study of cinema and its narrative components, did not construct any comprehensive model; neither did they rigorously compare language as a cinematic system [2]. They advanced considerations such as Fabula: "the set of events tied together which are communicated to us in the course of the work, what has in effect happened", and Sjuzet [22]; "how the reader becomes aware of what happened"," the order of appearance (of the events) in the work itself" [22], as of prime importance in the understanding of narrative. The macro structural approach advanced by Propp [23] has been applied in AI community as a tangible model for the development of storytelling systems [12].

Chatman [24] however, suggests that the structure of this model was in fact determined before the formulation of its rules. He also argues against the narrative universality of macro structural models. It is not clear for example that Propp's model applies to soap operas in the way it does to Russian fairy stories, or indeed that it would apply to the myths and fairy-stories of non-European cultures such as the Chinese. The French structuralists later explored the structures of narrative based on a linguistic approach. Works by Greimas, Todorov and especially Barthes $[25,17,1]$ 
can be cited here. However the applicability of structuralist theories, presents many difficulties to AI and VR, principally due to their very high level of abstraction. Analysis of structure seems inescapably tied to a view of narrative-as-artefact which seems to conflict with VR's real time process based narrative approach.

It appears that, the analytical benefits of such approaches being evident, it is however questionable to consider their use in the process view of building a narrative as VR regards it. The interaction with the user being of prime importance in VR applications, it seems that a process approach to narrative, based on character interaction (among them the user) would be more suitable and appropriate. A similar approach made by Heath [26], argued that, when considering the relation between subject and text, the narrative organisation of images and representations maintained the subject in position within the text. The subject is caught up by the text and bound not into position but into the process of narrativisation, the text moving the subject in a constantly shifting regulation and containment [27]. The consideration of "narrativisation" and the role of the subject bringing there similarities within the "storification" process and the role of the user in Aylett's approach [28].

\subsection{Conclusion}

Both the mimetic and diegetic approaches to narrative considered here lead to the conclusion that none of them seems to be directly applicable to VR. It is our belief that a process view of story, as opposed to a chronological view of narrative, should be adopted in order to provide VR with a more participative narrative form that can be drawn upon from both mimetic and diegetic considerations. 


\section{A PARTICIPATORY FORM OF NARRATIVE}

\subsection{Introduction}

So far we have identified several key issues concerning narrative in VR. We argue that narrative forms within VR should not be approach from a structuralist or analytical angle but should instead consider process-based or emergent theories. Drawing on VR characteristics of immersion and interactivity, we also see a need for such a narrative model to be particularly sensitive to questions of believability and the role of the user. Contrary to other narrative media already mentioned, users in a 3D virtual environment play a central part in the building of the story and their own overall experience since this depends upon their actions, reactions and behaviour within the world itself. It is for this reason that a character-centred approach seems preferable to an Aristotelian plot-centred one. We argue that it is important, given the freedom that VR potentially offers to the user, to present a model that supports a nonrestrictive and flexible approach to any possible plot development.

While we have failed to find the elements for such a model in the media considered above, we believe that there are other narrative forms much less frequently studied which may be more applicable. Models that are based primarily on the user or spectator's experience rather than proceeding from an authorial-based perspective might offer an alternative to the classical but inappropriate narrative forms studied so far. Participative models might also offer a different approach to the management of real time within narrative construction. By proposing adequate means of communication in accordance with 3D Virtual Environments the immersed user might 
exploit the characteristics of real time in the dynamic process we call "storyfication". An evaluation of such participative narrative media can then help in identifying key elements towards a narrative theory of VR.

\subsection{Participative narrative forms}

Participative forms of narrative have already demonstrated their potential through forms such as Live Role Playing Games (LRPG), Interactive and Improvisational theatre (IT/IMPROV) and to some extent in live historical re-enactment. It is immediately clear that in the case of IT, the constraints in place on the actors and the audience present a number of similarities with VR. In IT, the actors are usually given a certain amount of information interactively by the audience, and then act "in character"; applying this material creatively. Narrative emerges through the interaction between the different actors, who may themselves be advised by a part of the audience. A more structured version of this approach can be seen in Forum Theatre [5]. This allows sections of the audience to halt the action in order to provide new guidance to an actor, or indeed allows an actor to halt it if he or she cannot continue in role without further information. Boal coined the term 'spectACTOR' for the role played by audience members in this process to emphasise the difference from passive reception.

The existence of such approaches conflicts directly with the suggestion that narrative depends on the difference between authoring time and presenting time, making emergent narrative a contradiction in terms. This conception suggests that narrative can only exist if it can be defined as artefact, essentially the output of the authoring 
process. The process view, in opposition to this, defines narrative as the internal result of the human 'storification' process, which may be activated for someone playing the traditional god-like authorial role, the traditional passive spectator role, or in many combinations of these opposed roles. We argue that emergent narrative forms and practices in such participative narratives offer a more appropriate approach to VR than classical approaches [28]. The role of the audience in IT could be easily assimilated to the role played by the user in a VR application. Despite the fact that believability and certain behavioural considerations have to be dealt with, the actors acting reactively to the audience's interventions - could also be relatively accurately modelled.

Participative forms such as IT defy in some ways the usual conception of narrative as a storyline moving from pre-defined plot points in order to form a coherent, interesting and entertaining narrative experience. This does not mean however that no control at all is exercised over the direction in which narrative is developed. In IT, control is distributed between actors who use their dramatic experience - and possibly some pre-narrative discussion or general policy - to make choices, which result in an interesting narrative experience. Thus the authorial role alters in content and may no longer be centralised in one person. On the other hand, the role of a Game-Master in a LRPG does centralise these responsibilities, though it by no means excludes the initiative or participation of the role-players themselves. In order to evaluate this guiding semi-authorial function, it becomes necessary to consider deep knowledge elicitation of professionals and/or qualified people in the participative media of IT, IMPROV and LRPG. We are carrying out this work currently and results will be the subject of later publication. 
The control operated by the LRPG Game-Master or the IT actors on the unfolding of a narrative seems - this is an early statement - to consider primarily the entertainment values of events or actions rather than a pre-established plot line. Providing the spectator/actor or player with a structure that takes into account their level of satisfaction and enjoyment regarding the "in display" or emergent narrative takes precedence over a chronological pre-defined story exactly as intended by the author. In the case of LRPGs, which take place episodically over long elapsed periods of time (more than a year for example in some cases), the Game-Master usually maintains a high-level 'campaign-plan' which provides an abstract narrative structure. However our research suggests that this is quite flexible in the face of more interesting directions emerging from the role-players themselves.

By assessing the satisfaction of the spectator/actor as a key factor and by considering it as an articulation around which the unfolding of the narrative takes place, such models if correctly conducted cannot fail to provide the spectator with an entertaining and unique narrative experience. Both media are in form superficially dissimilar to VR with imagined locations and generally very few - if any - props. However this suggests the possibility of connecting a user friendly and satisfying narrative to its visually immersive representation, which would offer a unique narrative form, and thus the exploitation of VR's entertainment potential. 


\subsection{A dynamic systems view of narrative}

As an alternative to authorial and structural narrative approaches, we have proposed a process view of narrative as a dynamic system building itself from the interactions of its own narrative elements and factors. The consideration of the user and his or her behaviour as a primary resource for the storytelling system, brings a different perspective to the role of the user within the story, a character based interactive storytelling system. User and character together should provide, under the supervision of a "Drama manager", the material needed for the formation, development and unfolding of the narrative.

The idea of a story manager is not new in AI and VR [6], however we propose a somewhat different approach. Previous drama managers generally assessed the state of a story along one or several possible storylines and redirected it differently according to past events by introducing and running pre-defined story elements. Our approach to a story manager is motivated by the examples above. The LRPG GameMaster does not in fact intervene in the course of the narrative in the same way existing story managers propose. The Game-Master 's intervention criterion is what is satisfactory for the players rather than what plot element comes next. The distinction between the two is may not be evident in the sense that the goal of the story manager is, by providing the users with an interesting story, to satisfy them on a narrative plan.

Where the LRPG-based drama manager we are proposing differs from classic "plot" based drama manager, is that if, for instance, the user is clearly enjoying the performance of a certain task, that the task is completed is not a reason to stop the 
entertainment by forcing the user to move on the story line. The users should have the freedom to actually make the choice themselves; regarding a reasonable time limit, on whether they want to move on to the story line and when. It is our belief that the role of the drama manager should only to intervene in order to regulate the dramatic interest of the narrative, directing the narrative flow for this purpose but not imposing it upon the users.

A process view of narrative replaces the concept of narrative-as-artefact and the analytic approach to theory which goes with it with narrative-as-process and a synthetic approach to theory. It is then interesting to consider what existing processbased theories might be applicable to a narrative process. We have not so far investigated the possibilities in depth, but two possibilities immediately occur. In the first, the application of process algebras might provide a compositional framework within which characters are combined and these interactions produce changed characters and further interactions. By matching the interaction history against significant fragments expressed in process algebra [31] and rated for user interest, new processes and characters could be introduced as part of the management of the emerging narrative. It is clear that every narrative forms a closed system defined by the locations, the characters and their action repertoire and the external events that can occur. One of the important functions of dramatic management is to trigger external events (at its most simple, the 'wandering monsters' of RPG dungeon fame), add to subtract from the action repertoire of characters, and add or remove characters from the narrative world. 
A second and more encompassing theoretical approach might borrow from dynamic systems theory [32] in which methods of describing the possible trajectories of the dynamic system over a multi-dimensional surface are sought. This intuitively corresponds to the idea that a narrative generated dynamically by interaction represents a trajectory across the story-surface of all possible interactions. An element of this approach can be seen in Cavazza $[29,30]$ in which a universal plan [33] for each character is used as a description of the narrative space. The role of story management in this view is to guide and evaluate the trajectory. This account potentially offers its own way of evaluating the direction of narrative interaction. If one extracts a number of key dimensions from the imagined surface, it is then possible to visualise properties such as the rapidity of change along those dimensions for particular interactions.

For example, causality may be considered an important component of narrative. In some parts of the imagined story surface, an event may generate few consequences, while on other parts of the surface; an event may generate many consequences. One can visualise this as an area of relative flatness in the first case, or of steep gradient in the second. The role of dramatic management is then to navigate over the surface such that the gradient changes in an 'interesting' way', effectively producing something like a narrative roller coaster ride (though not necessarily with as many ups and downs as a real-world roller-coaster). The concept of dispatchers quoted by Cavazza $[29,30]$ and based on Barthes one then be one factor in such dramatic management. 
The story-surface approach currently seems a promising one, but clearly requires much more formalisation in order to be made operational. Explicit representation of the whole surface is unlikely to be feasible except in stricly-limited cases, so that representational and navigational issues will have to be dealt with.

In this view of narrative, the artefact dealt with by earlier theorists overlaps completely in time with its display rather than preceding it. An objection may then be that locally generated interaction gives no guarantee of the quality of the final product - narrative as artefact - from a global perspective. However what one has to remember here is the very different role of what had been the audience. By participating, the user commits to the narrative in a way that a spectator cannot, and rather than evaluating as detached observer, acts as engaged character. Thus their personal trajectory is as different in nature from that of a spectator as participating in a train crash is from reading about it on the news later. As is said of some anecdotes intended to amuse 'you had to be there'. 'Being there' is in our view the defining characteristic of VR.

\section{CONCLUSION}

In this paper, we considered arguments for a different conception of narrative as a process and expressed our belief that a narrative theory proper to VR should borrow from narrative media where interaction is predominant. Current narrative models used within the AI community prove to be problematic regarding their applicability to an interactive virtual environment. This paper presented a methodological approach to the narrative question within VR and aimed at highlighting the needs for the AI community to consider a narrative form appropriate to the particular characteristics of 
the medium. A narrative form where, interactivity as well as user satisfaction, constitute a basis for the construction and unfolding of a narrative, flexible enough in its articulation, to bring maximum satisfaction to the user (i.e. in terms of experience, enjoyment and interest). For this reason, interactive, improvisational theatre or Live Role Playing Games appear to us a reasonable and tangible source of information. 


\section{REFERENCES}

[1] Barthes R. Communication 8, Introduction à l'analyse structurale des récits. 1966. Editions du Seuil. 1981

[2] Bordwell D. Narration in the fiction film. Routledge. London. 1986

[3] Metz C. La Grande syntagmatique du film narratif. In: L'analyse structurale du récit. Communication 8. Points. 1966

[4] Bordwell D. Some theories of narration. In: Narration in the fiction film. Routledge. London. 1986

[5] Boal, A. Legislative theatre: Using performance to make politics. Routledge. 1999

[6] Mateas M. Carnegie Mellon University - An Oz - centric review of interactive drama and believable agents. 1997; CMU-CS-97-156

[7] Mateas M, Sengers P. Carnegie Mellon University - Narrative Intelligence. 1999; AAAI Fall Symposium on Narrative Intelligence

[8] Mateas, M. Carnegie Mellon University - A preliminary poetics for interactive drama and games. 2001; Proceedings of SIGGRAPH 2001.

[9] Bates J. Carnegie Mellon University - The nature of characters in interactive worlds and the Oz project. 1992; CMU-CS-92-200

[10] Bates J, Loyall B / Reilly S. Carnegie Mellon University - An architecture for action, emotion and social behaviour. 1992; CMU-CS-92-144

[11] Goldberg A, Perlin K. Media Research Laboratory, New York University IMPROV: A system for scripting interactive actors in virtual worlds. 1996; Proc. Siggraph 96, H. Rushmeier, ed., ACM Press, NewYork, 1996, pp. 205-216

[12] Brna, Paiva and al, Teatrix, NIMIS project.. 
[13] Hayes-Roth B. Stanford University, Knowledge Systems Laboratory - Agents of stage: Advancing the state of the art of AI. 1995; Report no KSL-95-50.

[14] Tolkien JJR. The lord of the rings. Harper \& Collins 2002

[15] Plato. La republique. GF-Flammarion, Paris 1966.

[16] Aristotle. The Poetics of Aristotle, translation and commentary by Stephen Halliwell 330 B.C. Duckworth, 1987.

[17] Todorov T. Grammaire du Decameron. 1970. The Hague. 1970.

[18] Aylett, R. Narrative in virtual environments - towards emergent narrative. 1999; AAAI Symposium on Narrative Intelligence.

[19] Nath, S. Central Saint Martin's College - Emotion Based Narratives. 2001; MA thesis of Art and Design. 2001.

[20] Dautenhahn K. University of Hertfordshire - Story-telling in virtual environments. Intelligent Virtual Agents Workshop, 13th European Conference on Artificial Life, 1998.

[21] Bordwell D. Diegetic theories of narration. In: Narration in the fiction film. Routledge. London. 1986

[22] Tomashevsky, B. Thematique. In Todorov, Theorie de la literature. Paris. 1966.

[23] Propp V. Morphology of the Folktale. 1928. University of Texas Press, 1998

[24] Chatman S. Story and Discourse, narrative structure in fiction and film.1978. Cornell paperbacks, Cornell University Press. 1989.

[25] Greimas AJ. Elements pour une theorie de l'interpretation du recit mythique. In: Introduction a l'analyse structurale des recits. Communications 8. Points. 1966 
[26] Heath S. Question of Cinema. London: Macmillan 1981

[27] Lapsey R, Westlake M. Film Theory: an introduction. Manchester University Press 1988

[28] Louchart S, Aylett R University of Salford - Narrative theories and emergent interactive narrative. NILE02. 2002; 1-8

[29] Cavazza M, Charles F, Mead S. University of Teesside - Characters in search of an author: AI-based Virtual Storytelling. 2001; IVA 2001: 156-170

[30] Cavazza M, Charles F, Mead S. University of Teesside - Agent's interaction in virtual storytelling.2001; International Conference on Virtual Storytelling 2001: 145 154

[31] Fokkink WJ. Introduction to Process Algebra, In Texts in Theoretical Computer Science, An EATCS Series. 2000; Springer-Verlag, 2000.

[32] Luenberger D. Introduction to Dynamic Systems: Theory, Models, and Applications. 1979. Wiley 1979

[33] Schoppers M. Advanced Decision Systems - Universal plans of reactive robots in unpredictable environments. 1987; Proceedings of IJCAI 1987 
